\title{
RESISTENCIA ANTIMICROBIANA DE Staphylococcus aureus AISLADO EN QUESOS FRESCOS ARTESANALES ELABORADOS EN ZONAS RURALES DE RIOBAMBA-ECUADOR
}

\section{Antimicrobial Resistance of Staphylococcus aureus Isolated In Fresh Handmade Cheeses Made In Rural Zones of Riobamba-Ecuador}

${ }^{1-2}$ Ana Albuja Landi* ${ }^{1-2}$ Sandra Escobar Arrieta, ${ }^{2}$ Luis Guevara Iñiguez, ${ }^{3}$ Félix Andueza Leal, 'Pamela Yugcha Pilamunga, ${ }^{1}$ Paola Arguello Hernández

${ }^{1 S}$ eguridad Alimentaria Grupo de Investigación y Desarrollo "SAGID”, Escuela Superior Politécnica de Chimborazo, Facultad de Ciencias, Riobamba, Ecuador

${ }^{2}$ Leishmaniosis y otras Parasitosis en el Ecuador "LEISHPAREC", Escuela Superior Politécnica de Chimborazo, Facultad de Ciencias, Riobamba, Ecuador

${ }_{3}$ Universidad Central del Ecuador, Quito, Ecuador

*aalbuja@espoch.edu.ec

\section{$R$ esumen}

La investigación tuvo por objeto evaluar la calidad microbiológica de los quesos frescos artesanales mediante el recuento de Staphylococcus aureus y aislar cepas de este microorganismo, para medir su resistencia a los antibióticos. Las queseras artesanales intervenidas están ubicadas en áreas rurales de Riobamba-Ecuador (San Juan, Pungalá y Punín). El proceso de muestreo se llevó a cabo de acuerdo con la normativa nacional (NTE INEN 1529-2-2013) y para la cuantificación de Staphylococcus aureus se siguió la Norma NTE. INEN 1528-2012. El aislamiento e identificación de las cepas bacterianas se realizó mediante técnicas microbiológicas, corroborando estos resultados con pruebas bioquímicas. Para determinar la susceptibilidad antimicrobiana de cepas aisladas de Staphylococcus aureus se utilizó la prueba de difusión en disco de Kirby-Bauer. La prevalencia de Staphylococcus aureus fue de $83.33 \%$, mismos que presentan resistencia a penicilina $(100 \%)$, cefoxitina $(33,33 \%)$ y eritromicina $(80 \%$ resistencia intermedia). Estos resultados muestran que los quesos frescos artesanales analizados superan los rangos de referencia nacionales y que los altos niveles de resistencia a los antibióticos constituyen un potencial problema de salud pública.

Palabras claves: resistencia antimicrobiana, queso fresco artesanal, Staphylococcus aureus.

\section{A bstract}

The aim of this research was to evaluate the microbiological quality of fresh handmade cheeses through the quantification of Staphylococcus aureus and, isolate theirs strains from these cheeses for measuring their resistance to antibiotics. The handmade cheese factories where the samples were taken are located in rural areas of Riobamba-Ecuador (San Juan, Pungalá and Punín). The sampling process was carried out based on the national regulation (NTE INEN 1529-2-2013), and the national regulation NTE. INEN 1528-2012 was used for the quantification of these microorganisms. The isolation and identification of the bacterial strains were performed through microbiology techniques, 
these results were corroborated with biochemical tests. The Kirby-Bauer disk diffusion test was used to determine antimicrobial susceptibility of isolated Staphylococcus aureus strains. Prevalence of Staphylococcus aureus was $83.33 \%$. They were resistant to Penicillin (100\%), Cefoxitin (33.33\%) and Erythromycin (80\% intermediate resistance). These results showed that the fresh artisanal cheeses analyzed exceeded the national reference levels and, the high levels of resistance to antibiotics are a potential problem in public health safety

Keywords: antimicrobial resistance, fresh artisanal cheese, Staphylococcus aureus.

\section{INTRODUCCIÓN}

Staphylococcus aureus son bacterias causantes de mastitis bovina, han sido identificadas en animales, queseras artesanales, manipuladores, superficies de contacto, leche cruda y en el queso, es decir pueden transmitirse a lo largo de la cadena alimentaria. (1)

La calidad microbiológica y la seguridad del queso comienzan con la leche. Por su abundancia de nutrientes y $\mathrm{pH}$ casi neutro, la leche sirve como un medio de crecimiento y desarrollo de microorganismos contaminantes, asociados con el proceso de manipulación y condiciones higiénico sanitarias deficientes, principalmente en los establecimientos artesanales, ubicados en zonas rurales, que no tienen implementadas prácticas correctas de higiene.

Es muy común encontrar en el queso fresco artesanal la presencia de Staphylococcus aureus, por la utilización de leche proveniente de ganado vacuno enfermo con mastitis o por encontrarse en la piel y mucosas del ser humano que puede transmitirse a los alimentos por una mala manipulación. (2) Staphylococcus aureus multirresistente surgió hace décadas debido al uso inadecuado de antibióticos en el ganado. (1)

La resistencia a los antibióticos aumenta el costo del tratamiento de las infecciones y puede ser la causa del fracaso terapéutico y la propagación de la resistencia antibiótica a patógenos humanos y animales. Se cree que la cadena alimentaria es una de las principales vías por las que se extiende dicha resistencia. El queso es un producto que posee una microbiota natural que combinada con una serie de microorganismos ambientales y los provenientes de la mala higiene en la manipulación, son actores clave en la transmisión de la resistencia a los antibióticos entre bacterias benéficas y patógenas. (3)

El presente trabajo se realizó para determinar la resistencia antimicrobiana de cepas de Staphylococus aureus aisladas en quesos frescos artesanales elaborados en zonas rurales de Riobamba, Ecuador.

\section{MATERIALES Y MÉTODOS}

Se trabajó con muestras de queso fresco provenientes de seis queseras artesanales de las parroquias rurales de San Juan, Pungalá y Punín, de cada quesera se tomaron 2 muestras por un periodo de tres semanas aleatorias (36 muestras) siguiendo lo especificado por la Norma NTE. INEN 1529-2-2013. (4)

\section{MÉTODOS DE ANÁLISIS}

\section{Aislamiento microbiano de Staphylococcus aureus}

Para el recuento de Staphylococcus aureus se prepararon diluciones con agua peptonada estéril al $0,1 \%$ a partir de la solución madre (homogenizar 10 gramos de queso fresco con $90 \mathrm{ml}$ de diluyente) se prepararon diluciones seriadas hasta $10^{-5}$. Se sembró $1 \mathrm{ml}$ de la dilución en Placas Petri Film Staph Express 3M, mismas que se incubaron a $35^{\circ} \mathrm{C}$ durante 3 horas; identificando colonias rojo-violetas en la placa (5). Se sembró como control en Agar Manitol Salado, en el que se aislaron colonias ca- 
racterísticas fermentadoras de azúcares, se hicieron repiques de aislamiento para purificar las cepas, todos los aislamientos fueron corroborados con Tinción Gram, prueba de catalasa y coagulasa, según lo establecido en la Norma INEN 1529-14-98

\section{Pruebas de susceptibilidad microbiana}

Una vez aisladas las cepas de $S$. aureus, se procedió a determinar la prueba de susceptibilidad para lo cual se utilizó el método de difusión de disco en agar Kirby-Bauer (6), se ensayó la resistencia/sensibilidad con discos de: penicilina PE 10 unid, Cefoxitina FOX 30 ug, Tetraciclina TE 30 ug, Amikacina AK 30 ug, Vancomicina VA, Ciprofloxacina CIP 5 ug, Clindamicina DA 2 ug y, Eritromicina $\mathrm{E} 15 \mathrm{ug}$. Se midieron los halos de inhibición $y$ el criterio de sensibilidad o resistencia a cada agente se determinó según las especificaciones del CLSI (2015) (7), observadas en la tabla 1.

\begin{tabular}{|l|c|c|c|c|}
\hline \multicolumn{1}{|c|}{ Antibiótico } & $\begin{array}{c}\text { Concentración } \\
(\boldsymbol{\mu g})\end{array}$ & $\begin{array}{c}\text { Resistente } \\
(\mathbf{m m})\end{array}$ & $\begin{array}{c}\text { Intermedio } \\
(\mathbf{m m})\end{array}$ & $\begin{array}{c}\text { Susceptible } \\
(\mathbf{m m})\end{array}$ \\
\hline Penicilina & 10 (unidades) & $\leq 28$ & - & $\geq 29$ \\
\hline Tetraciclina & 30 & $\leq 14$ & $15-18$ & $\geq 19$ \\
\hline Clindamicina & 2 & $\leq 14$ & $15-20$ & $\geq 21$ \\
\hline Eritromicina & 15 & $\leq 13$ & $14-22$ & $\geq 23$ \\
\hline Ciprofloxacina & 5 & $\leq$ & $16-20$ & $\geq 21$ \\
\hline Vancomicina & - & - & - & $\geq 21$ \\
\hline Amikacina & 30 & $\leq 14$ & $15-16$ & $\geq 17$ \\
\hline Cefoxitina & 30 & $\leq 21$ & - & $\geq 22$ \\
\hline
\end{tabular}

Tabla 1. Criterios de interpretación para el método de difusión del disco para Staphylococcus aureus según el CLSI 2015

\begin{tabular}{|c|c|c|}
\hline Procedencia & Quesera & UFC/g \\
\hline San Juan A & 1 & $1110 \pm 442$ \\
\hline San Juan B & 2 & $4487 \pm 3796$ \\
\hline Quimiag C & 1 & $3050 \pm 2925$ \\
\hline Quimiag D & 2 & $600 \pm 529$ \\
\hline Pungalá E & 1 & $67 \pm 115$ \\
\hline Pungalá F & 2 & $4470 \pm 4314$ \\
\hline
\end{tabular}

Valores de referencia de Staphylococcus aureus (INEN 1528:2012 - MAX. 102 UFC/g )

Tabla 2: Recuento de Staphylococcus aureus

\section{DISCUSIÓN DE RESULTADOS}

Los recuentos microbianos para Staphylococus aureus (tabla 2), indican que el 83,3\% (prevalencia) de muestras analizadas no cumple con los parámetros microbiológicos establecidos para este microorganismo según la Norma NTE INEN 1528:2012 Quesos frescos no maduros Requisitos (8). Como han descrito otros autores, la presencia de Staphylococus aureus en los quesos artesanales es un indicativo de contaminación de la materia prima, proceso de producción y la manipulación (9). El aislamiento de Staphylococus aureus, (tabla 3), fue confirmado por la fermentación en agar manitol (medio selectivo), y las pruebas bioquímicas de coagulasa y catalasa. (10)

La contaminación por Staphylococus en los quesos puede ocurrir por prácticas de fabricación inadecuadas, deficientes condiciones de almacenamiento, materia prima contaminada y por las características físico químicas del queso que lo hacen un medio favorable para el crecimiento de estos microorganismos. (11) En la tabla 2 se observa que en los recuentos de Staphylococcus aureus, las desviaciones son amplias, debido a que los muestreos fueron aleatorios, considerando que las queseras artesanales no cuentan con procedimientos operativos estandarizados y que los manipulados son el principal vehículo de contaminación de este microorganismo, los resultados fueron muy diferentes entre un muestreo y otro.

La prevalencia de Staphylococcus aureus en los quesos analizados fue del $83.33 \%$, datos similares a los reportados por Mehli L. de la Universidad de Norweglan 2016 (1), que detecta cepas de $S$. aureus en el $45 \%$ de muestras analizadas de producción artesanal (suero, leche, queso) y de los 72 aislamientos se encontró 16 perfiles diferentes de enterotoxinas, siendo esta la causa de intoxicaciones alimentarias, por lo que los 
quesos analizados en esta investigación podrían ser una amenaza para la salud del consumidor.

Varios autores concuerdan que en la elaboración de quesos artesanales existen parámetros críticos como la escasa calidad sanitaria de la leche cruda, la deficiencia de prácticas correctas de ordeño (vacas con mastitis) sumado a la mala manipulación y la falta de prácticas de higiene hace de este producto un riesgo sanitario. $(2,12,13)$

En tres muestras de queso (tabla 3), se presentan casos de $S$. aureus coagulasa negativa, siendo estas cepas las más dañinas debido a la capacidad de producción de enterotoxinas termoestables con actividad antigénica en diversas matrices de alimentos especialmente en productos lácteos como los quesos frescos (11), pudiendo ser el consumo de estos quesos artesanales un potencial peligro de toxiinfecciones alimentarias.

En los aislamientos realizados se encontró cepas de S. aureus $100 \%$ resistentes frente a penicilina y un $80 \%$ de resistencia intermedia frente a eritromicina (tabla 4); datos que concuerdan con el estudio de Flórez A, 2015 en el Instituto de Productos Lácteos de Asturias, quién reporta comunidades bacterias entre ellas $S$. aureus resistente a la eritromicina y tetraciclina en quesos (3), siendo un microorganismo que expresa varios factores de virulencia como toxinas, enzimas, adhesinas y proteínas de superficie que permiten a este patógeno su capacidad de evolución y adaptación a diferentes entornos (14).

Varios autores concuerdan en que la penicilina es el fármaco más utilizado en el tratamiento de la mastitis bo-

\begin{tabular}{|c|c|c|c|c|}
\hline Muestra* & $\begin{array}{c}\text { Crecimiento } \\
\text { en agar } \\
\text { manitol }\end{array}$ & $\begin{array}{c}\text { Tinción } \\
\text { Gram }\end{array}$ & $\begin{array}{c}\text { Prueba } \\
\text { Catalasa }\end{array}$ & $\begin{array}{c}\text { Proeba } \\
\text { Coagulasa }\end{array}$ \\
\hline 1A & $(+)$ & $(+)$ & $(+)$ & $(+)$ \\
\hline $2 \mathrm{~A}$ & $(+)$ & $(+)$ & $(+)$ & $(+)$ \\
\hline 3A & $(+)$ & $(+)$ & $(+)$ & $(+)$ \\
\hline 1B & $(+)$ & $(+)$ & $(+)$ & $(+)$ \\
\hline 2B & $(+)$ & $(+)$ & $(+)$ & $(+)$ \\
\hline 3B & $(+)$ & $(+)$ & $(+)$ & $(+)$ \\
\hline 1C & $(+)$ & $(+)$ & $(+)$ & $(+)$ \\
\hline 2C & $(+)$ & $(+)$ & $(+)$ & $(+)$ \\
\hline 3C & $(+)$ & $(+)$ & $(+)$ & $(+)$ \\
\hline 1D & $(+)$ & $(+)$ & $(+)$ & $(+)$ \\
\hline 2D & $(+)$ & $(+)$ & $(+)$ & $(+)$ \\
\hline 3D & $(-)$ & $(+)$ & $(-)$ & $(-)$ \\
\hline 1E & $(+)$ & $(+)$ & $(+)$ & $(+)$ \\
\hline 2E & $(-)$ & $(+)$ & $(-)$ & $(-)$ \\
\hline 3E & $(-)$ & $(+)$ & $(-)$ & $(-)$ \\
\hline 1F & $(+)$ & $(+)$ & $(+)$ & $(+)$ \\
\hline 2F & $(+)$ & $(+)$ & $(+)$ & $(+)$ \\
\hline 3F & $(+)$ & $(+)$ & $(+)$ & $(+)$ \\
\hline
\end{tabular}

* la codificación corresponde: el número a la semana de muestreo y la letra a la quesera muestreada por duplicado

Tabla 3. Identificación de Staphylococcus aureus en muestras de queso fresco artesanal

\begin{tabular}{|l|c|c|c|c|c|c|c|c|c|c|c|c|c|c|c|c|c|c|cc|c|}
\hline $\begin{array}{c}\text { Muestra / } \\
\text { antibiótico }\end{array}$ & 1A & 2A & 3A & 1B & 2B & 3B & 1C & 2C & 3C & 1D & 2D & 3D & 1E & 2E & 3E & 1F & 2F & 3F & $\begin{array}{c}\text { \% } \\
\text { resistencia }\end{array}$ & $\begin{array}{c}\text { \% suscepti- } \\
\text { bilidad }\end{array}$ \\
\hline Penicilina & R & R & R & R & R & R & R & R & R & R & R & & R & & & R & R & R & 100,0 & 0 \\
\hline Tetraciclina & S & S & R & S & S & S & S & R & R & S & S & & R & & & S & R & R & 33,33 & 66,67 \\
\hline Clindamicina & S & S & S & S & S & S & S & S & S & S & S & & R & & & S & R & R & 13,33 & 86,67 \\
\hline Eritromicina & S & S & S & S & S & S & S & S & S & S & S & & R & & & S & R & S & 6,67 & 93,33 \\
\hline Ciprofloxacina & S & S & S & S & S & S & S & S & S & S & S & & R & & & R & S & S & 0,00 & 100 \\
\hline Vancomicina & S & S & S & S & S & S & S & S & S & S & S & & R & & & S & S & S & 0,00 & 100 \\
\hline Amikacina & S & S & S & S & S & S & S & S & S & S & S & & R & & & S & R & R & 20,00 & 80 \\
\hline Cefoxitina & I & I & S & I & I & S & I & I & S & I & I & & I & & & I & I & I & 80,00 & 20 \\
\hline
\end{tabular}

S: susceptible

$\mathrm{R}$ : resistente

I: intermedio 
vina y además es el que más resistencia presenta (15), de ahí el riesgo que conlleva el consumo de alimentos contaminados con bacterias patógenas resistentes. La resistencia microbiana se traduce en la escasez de terapias antimicrobianas, aumentando el costo del tratamiento e incluso la causa de la falla terapéutica y de allí la existencia de bacterias intratable. (3)

En otro estudio similar realizado en quesos artesanales de Brasil, encontraron cepas de Staphylococus multirresistentes a agentes antimicrobianos como B-lactámicos, vancomicina y linezolida, fármacos con propiedades terapéuticas de importancia en medicina humana y veterinaria, constituyendo esta matriz de alimentos un reservorio de resistencia microbiana (11).

Según Jordá G, 2012 en un estudio sobre la sensibilidad a los antibióticos mostró que todos los aislamientos de $S$. aureus en manipuladores de alimentos, presentaron resistencia a la clindamicina, meticilina y eritromicina (10), coincidiendo que el manipulador es el portador del microorganismo causante de contaminación en el producto final.

Como han descrito otros autores, se hace necesaria la implementación de prácticas correctas de higiene en los establecimientos artesanales, normas básicas como el lavado frecuente de las manos y el uso de mascarillas, así como la pasteurización de la leche cruda y el control sani- tario del ganado vacuno, para garantizar la inocuidad del queso fresco y controlar la propagación de bacterias resistentes a los antibióticos. $(3,12,13)$

\section{CONCLUSIONES:}

Los quesos artesanales producidos en queseras rurales del cantón Riobamba ubicadas en Pungalá, San Juan y Punín no cumplen con los parámetros microbiológicos establecidos para Staphylococcus aureus en la Normativa INEN. La prevalencia de este microorganismo patógeno en los quesos analizados fue del $83.33 \%$; mismos que presentan resistencia a Penicilina 100,00\%; Cefoxitina $33,33 \%$ Clindamicina $20,00 \%$ y Eritromicina $80,00 \%$ de resistencia intermedia. Se recomienda que la Autoridad Sanitaria Nacional capacite al productor artesanal en la implementación de Prácticas Correctas de Higiene, para garantizar la inocuidad del queso fresco.

1. Mehli L, Hoel S, Thomassen GMB, Jakobsen AN, Karlsen H. The prevalence, genetic diversity and antibiotic resistance of Staphylococcus aureus in milk, whey, and cheese from artisan farm dairies. Int Dairy J [Internet]. 2017 [citado 15 Mayo 2018]; 65: 20-7. Disponible en: http://dx.doi.org/10.1016/j. idairyj.2016.10.006

2. Lemus D, Badaoui M, Hassoun M. Estafilococos oxacilino resistentes en queso blanco fabricado en el estado Anzoátegui, Venezuela. Revista de la Sociedad Venezolana de Microbiología [Internet]. 2008 [citado 24 de abril 2018]; 28: 48-54. Disponible en: http://www.scielo.org.ve/scielo.php?pi$\mathrm{d}=\mathrm{S} 131525562008000100010 \& a \mathrm{mp}$;scri $\mathrm{t}=$ sci_pdf\&amp;tlng=es

3. Flórez AB, Mayo B. Diversity and dynamics of antibiotic-resistant bacteria in cheese as determined by PCR denaturing gradient gel electrophoresis. Int J Food Microbiol. 2015; 214: 63-69.

4. NORMA NTE . INEN 1529-2-2013: Control microbiológico de los alimentos. Toma, envío y preparación de muestras para el análisis microbiológico

5. Silva B, Caraviello D, Rodrigues A, Ruegg P. Evaluation of Petrifilm for the isolation of Staphylococcus aureus from milk samples. Journal of dairy science [Internet]. 2005 [citado 2 de mayo 2018]; 88: 30003008. Disponible en: https://www.sciencedirect.com/science/article/pii/S0022030205729805

6. Bernal M, Guzmán M. El antibiograma de discotecas. Normalización de la técnica de Kirby-Bauer. Biomédica. 1984, 4:112-121. 
7. Jorgensen H, Turnidge D. Susceptibility test methods: dilution and disk diffusion methods. En Manual of Clinical Microbiology. 11 $1^{\mathrm{a}}$ ed. Washington:American Society of Microbiology; 2015. p. 1253-1273. 8. NORMA NTE. INEN 1528:2012 : Quesos frescos no maduros requisitos

9. Arguello P, Lucero O, Castillo G, Escobar S, Albuja A, Gallegos J, et al. Calidad microbiológica de los quesos artesanales elaborados en zonas rurales de Riobamba (Ecuador). Revista Perspectiva. 2015; 16: 65-74.

10. Jordá B, Marucci S, Guida M, Pires S, Manfredi A. Portación y caracterización de Staphylococcus aureus en manipuladores de alimentos. Rev. Argentina de Microbiología . 2012; 44: 101-104.

11. Nunes R, De Souza C, Pereira K, Del Aguila R. Identification and molecular phylogeny of coagulase-negative staphylococci isolates from Minas Frescal cheese in southeastern Brazil: Superantigenic toxin production and antibiotic resistance. Journal of dairy science [Internet]. 2016 [citado 19 de mayo 2018]; 99: 2641-2653. Disponible en: https://www.sciencedirect.com/science/article/pii/S0022030216000849

12. Martínez A, Villoch A, Ribot A, Ponce P. Evaluación de la calidad e inocuidad de quesos frescos artesanales de tres regiones de una provincia de Cuba. Rev. Salud Anim. 2013; 35: 210-213.

13. Castro G, Martínez E, Martínez R, Espinoza A. Caracterización de la microbiota nativa del queso Oaxaca tradicional en tres fases de elaboración. Rev Sociedad Venezolana de Microbiología. 2013; 33: 105-109. 14. Kumar K, Boutin A. Antibiotic resistance. Journal of infection and public health [Internet]. (2017) [citado 7 Mayo 2018]; 10: 369-378. Disponible en: http://dx.doi.org/10.1016/j.jiph.2016.08.007

15. Raspanti CG, Bonetto CC, Vissio C, Pellegrino MS, Reinoso EB, Dieser SA, et al. Prevalence and antibiotic susceptibility of coagulase-negative Staphylococcus species from bovine subclinical mastitis in dairy herds in the central region of Argentina. Rev Argent Microbiol [Internet]. 2016 [citado 5 Mayo 2018]; 48: 50-56. Disponible en: http://www.sciencedirect.com/science/article/pii/S0325754115001662Frieri M, 16. NORMA NTE. INEN 1529-14:98: Control Microbiológico de los alimentos. Staphylococcus aureus. Recuento en placa de siembra por extensión en superficie. 